\title{
Influence of weak magnetic fields treatment on photoluminescence of GaAs
}

\author{
S.M. Red'ko \\ V. Lashkaryov Institute of Semiconductor Physics, NAS of Ukraine, \\ 45, prospect Nauky, 03028 Kyiv, Ukraine \\ Phone: 38 (044) 525-94-64; e-mail: redko.fotya@gmail.com
}

\begin{abstract}
Long-term transformations in photoluminescence of GaAs single crystals treated with pulsed weak magnetic fields have been obtained. Treatments were performed in the multi-pulse $(B=60 \mathrm{mT}, f=10 \mathrm{~Hz}, \tau=1.2 \mathrm{~ms}, t=5 \mathrm{~min})$ regime. The defect structure transformations were inferred from the radiative recombination spectra within the range 0.6 to $2.5 \mu \mathrm{m}$ at 300 and $77 \mathrm{~K}$. A possible mechanism of the observed modifications related with electron-spin transformation is discussed.
\end{abstract}

Keywords: photoluminescence, weak magnetic field, impurity-defect composition.

Manuscript received 18.03.14; revised version received 31.07.14; accepted for publication 16.09.14; published online 30.09.14.

\section{Introduction}

It is known $[1,2]$ that weak magnetic fields (WMF) with Zeeman's energy $<10^{-4} \mathrm{eV}$ induce changes in the defect subsystem of solids in a non-trivial way. Treatments under these magnetic fields occur with low energy absorption, considerably smaller than $k T$ value at room temperature. Therefore, WMF-induced structural transformations can be attributed to spin-dependent processes in this defect structure. Due to these processes, destruction of non-equilibrium complexes and formation of a new defect subsystem occur. The use of WMF can be efficient and promising method for modifying the impurity-defect composition and structural parameters of device materials. Besides, further investigations in this direction are required to understand all the field-induced processes.

\section{Experimental}

Czochralski-grown GaAs samples with orientation (111) of the $n$-type doped with Te were objects of our researches. The WMF treatment pulse regime with the parameters $(B=60 \mathrm{mT}, f=10 \mathrm{~Hz}, \tau=1.2 \mathrm{~ms}, t=1,5$, $10,15 \mathrm{~min}$ ) was chosen for our experiments. The defect structure of our samples was studied using the photoluminescent (PL) method within the spectral range $\Delta h v=(0.50 \ldots 2.04) \mathrm{eV}$. We used a powerful lamp ПЖ100 as an exciting radiation source. Its emission spectrum was cut with corresponding filters to separate their radiation with $h v \geq 2.0 \mathrm{eV}$. Measurements of PL were carried out at 77 and $300 \mathrm{~K}$. All the measurements were repeated during several weeks after the WMF treatment to reveal the time-dependent features.

\section{Results and discussions}

The radiative recombination spectrum of GaAs crystals at room temperature consists of one band peaked at about $0.99 \mathrm{eV}$, which is related with $\mathrm{V}_{\mathrm{Ga}} \mathrm{V}_{\mathrm{As}}$ complex [3]. The PL spectrum at $77 \mathrm{~K}$ exhibits two well-resolved luminescence bands, at 1.02 and $1.22 \mathrm{eV}$. The first peak is attributed to $\mathrm{V}_{\mathrm{Ga}} \mathrm{V}_{\mathrm{As}}$ and the second one is assigned to $\mathrm{V}_{\mathrm{Ga}} \mathrm{Te}_{\mathrm{As}}$ complexes [3]. The normalized dependences of observed bands intensity as a function of time after various WMF treatments are shown in the figure. One can see that the defect subsystem evolution in the nearsurface layer of semiconductor material depends on the treatment regime. In particular, the treatment for $t_{1}=1 \mathrm{~min}$ results in inessential changes of PL spectra. 
Other regimes of WMF treatments result in reversible (at $t_{2}=5 \mathrm{~min}$ ) and non-reversible (at $t_{3}=10,15 \mathrm{~min}$ ) changes of radiative recombination spectra of GaAs. The most pronounced changes were detected at $300 \mathrm{~K}$ for the $0.99 \mathrm{eV}$ PL band. It is easy to see that variations in the band intensity are similar for all treatment regimes until reaching a 15-day term. After that, a sharp gradation in the PL intensity, depending on duration of WMF treatment, was observed. The PL measurements at $77 \mathrm{~K}$ are indicative of transformation in the defect structure of material under investigation, but no confirmation of room-temperature measurements was detected. The general features were presented: the treatment for $1 \ldots 5 \mathrm{~min}$ results in feeble changes, while the $10 \ldots 15$ min treatment leads to the sufficient ones. It should be noted that the WMF treatment led to different transformations of the observed peaks at $77 \mathrm{~K}$. It can be caused by character of chemical bonds in $\mathrm{V}_{\mathrm{Ga}} \mathrm{V}_{\mathrm{As}}$ and $\mathrm{V}_{\mathrm{Ga}} \mathrm{Te}_{\mathrm{As}}$ complexes.

Changes of band intensities were not synchronous, so by changes in only the non-radiative channel, it cannot be explained. Perhaps, the change of both radiative and non-radiative transition probabilities as well as the change in the concentration of recombination-active centers can be responsible for the observed transformation.

It is known that the layer thickness that provides the PL signal is inversely proportional to the absorption coefficient. In the studied spectral range for GaAs, with the extinction coefficient $k=0.298$, the absorption coefficient $\alpha$ can be determined using the expression:

$\alpha=\frac{4 \pi k}{\lambda_{0}}$,

where $\lambda_{0}$ is the wavelength of PL excitation. For $\lambda_{0}=0.620 \mu \mathrm{m}$ estimated $\alpha \sim 10^{4} \mathrm{~cm}^{-1}$. Thus, the thickness of the layer emitting PL $\sim 10^{-4} \mathrm{~cm}$. That is, we can analyze transformation of the defect structure in semiconductor material at this evaluated depth. The observed modification cannot be the reason for natural aging of GaAs samples. The reference sample was kept under the same conditions as the treated ones between measurements. The PL spectrum of the reference sample did not change, while modification of the treated ones was detected.

Let us try to give a physical explanation to the observed transformations. The effect of electromagnetic induction can appear in semiconductor materials under the WMF treatment. Its value can be estimated from the well-known relation $(\sim 5 \mathrm{mV})$ and, obviously, is not able to induce any structural modifications. Zeeman's effect and Lorentz's force in magnetic field cannot be the reasons for the observed features, too. Despite these, the WMF induced effect is present and needs to be explained. Spin-transformation related processes can determine the reaction of defects modification $[1,2,4]$. WMF causes reorientation of electron spin. It can result in weakening chemical bonds and destruction of the complex formed by this atom. That is, due to the WMF treatment, destruction of non-equilibrium vacancycontained complexes $\left(\mathrm{V}_{\mathrm{Ga}} \mathrm{V}_{\mathrm{As}}\right.$ and $\left.\mathrm{V}_{\mathrm{Ga}} \mathrm{Te}_{\mathrm{As}}\right)$ occurs. After that, isolated $\mathrm{V}_{\mathrm{As}}$ and $\mathrm{V}_{\mathrm{Ga}}$ appear in the bulk of gallium arsenide. These point defects have small lifetime and can take part in formation of other defect complexes or/and move to natural drain (semiconductor surface). According to [3], the centers of fast (non-radiative) recombination are related to isolated $\mathrm{V}_{\mathrm{Ga}}$ and $\mathrm{V}_{\mathrm{As}}$. So, long-term transformation of PL spectra can be related with vacancy fluxes. If a new defect state of semiconductor material is thermodynamically unstable, the reverse processes will be observed. But, if impurity and native atoms occupy more energy-favorable places, non-reversible changes of PL spectra will be observed.
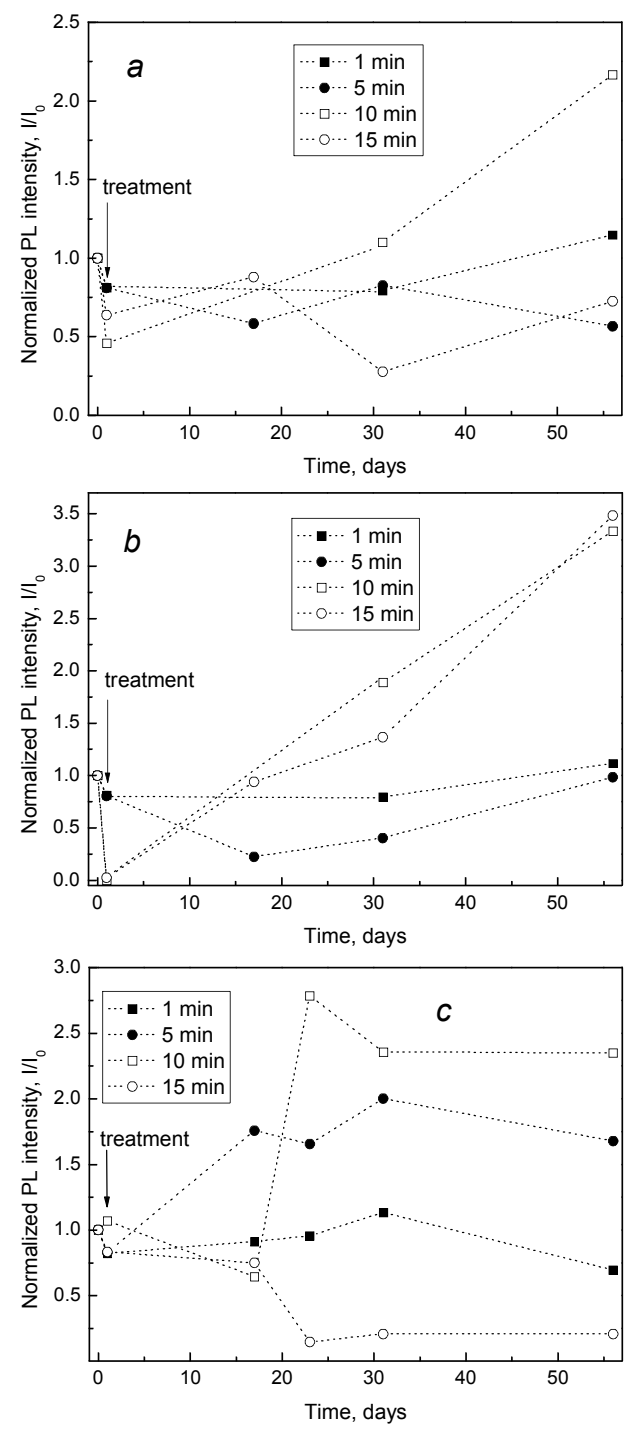

Fig. 1. Intensity of PL bands with peaks at $1.02 \mathrm{eV}, 77 \mathrm{~K}(a)$, $1.21 \mathrm{eV}, 77 \mathrm{~K}(b)$ and $0.99 \mathrm{eV}, 300 \mathrm{~K}(c)$ in GaAs crystals as a function of time after the WMF treatment. 


\section{Conclusions}

Analyzing the obtained data, it is possible to draw the following conclusions. The WMF treatment results in the change of local center concentrations that are responsible for both radiative and non-radiative recombination. Modification of the near-surface region of semiconductor material due to this treatment can be reversible or non-reversible. It is defined by the treatment regime. Radiative recombination bands associated with donor-acceptor pairs are the most sensitive to WMF treatments. It has been found that structural relaxation of semiconductor material is directly related with duration of WMF treatment. To ascertain micromechanisms of observable structural reorganization, additional researches are required.

\section{References}

1. Yu.I. Golovin, Magnetoplastic effects in solids // Physics of the Solid State, 46(5), p. 789-824 (2004).

2. R.B. Morgunov, Spin micromechanics in physics of plasticity // Phys. Usp. 47(2), p. 125-147 (2004).

3. K.D. Glinchuk, A.V. Prokhorovich, V.E. Rodionov, V.I. Vovnenko, The scheme of electronic transitions via $0.94,1.0,1.2$, and $1.3 \mathrm{eV}$ radiative centres in $\mathrm{n}$ GaAs // Phys. status solidi (a), 41(2), p. 659-668 (1977).

4. Ya. B. Zel'dovich, A. L. Buchachenko, E. L. Frankevich, Magnetic-spin effects in chemistry and molecular physics // Sov. Phys. Usp. 31(5), p. 385408 (1988). 